\title{
Multiple Successful Angioembolizations for Refractory Cardiac Failure in a Preterm with Rapidly Involuting Congenital Hemangioma
}

\author{
Amitava Sur, MBBS, DCH, DNB ${ }^{1,2}$ Heran Manraj, MD, FRCPC ${ }^{1,3}$ Pascal M. Lavoie, MD, FRCPC, PhD ${ }^{1,2}$ \\ Ken Lim, MD, FRCSC ${ }^{1,4}$ Douglas Courtemanche, MD, MS ${ }^{1,5}$ Paul Brooks, MBBS, FRACF ${ }^{1,6}$ \\ Susan Albersheim, MD, FRCPC, PhD ${ }^{1,2}$
}

${ }^{1}$ Children's and Women's Health Centre of British Columbia, Vancouver, Canada

2 Department of Pediatrics, University of British Columbia,

Address for correspondence Susan Albersheim, MD, FRCPC, PhD, Children's and Women's Health Centre of British Columbia, 4480 Oak Vancouver, Canada

${ }^{3}$ Department of Interventional Radiology, University of British Columbia, Vancouver, Canada

${ }^{4}$ Department of Maternal and Fetal Medicine, University of British Columbia, Vancouver, Canada

${ }^{5}$ Department of Plastic Surgery, University of British Columbia, Vancouver, Canada

${ }^{6}$ Department of Cardiology, University of British Columbia,

Vancouver, Canada

Am J Perinatol Rep 2016;6:e99-e103.

\begin{abstract}
Keywords

- $\mathrm{RICH}$

- high-output cardiac failure

- angioembolization

- preterm

Rapidly involuting congenital hemangiomas $(\mathrm{RICH})$ are the commonest variety of congenital hemangioma, often diagnosed antenatally as high-flow arteriovenous shunts causing hemodynamic compromise to the fetus. The postnatal management of such patients is often challenging. We present the case of an infant boy who was delivered prematurely at 29 weeks of gestation due to fetal compromise by a $\mathrm{RICH}$, with features of high-output cardiac failure and major systemic hemodynamic steal from peripheral organs. Two early angioembolizations were required to manage his high-output cardiac failure. To our knowledge, this infant is the smallest and earliest newborn case of successful angioembolization for a complex, life-threatening vascular anomaly. We discuss the interventional dilemmas regarding the optimal timing of delivery and early embolization.
\end{abstract}

Congenital hemangiomas (CHs), unlike the infantile variants, are present at birth and are typically violaceous, telangiectatic, or bossed lesions. They are categorized into two main subtypes based on their natural history of evolution: rapidly involuting $\mathrm{CH}$ (RICH) and noninvoluting $\mathrm{CH}^{1}{ }^{1} \mathrm{CHs}$ are differentiated from infantile hemangiomas angiographically by large and irregular feeding arteries, direct arteriovenous shunts, and intravascular thrombi. In the antenatal period, a fast flow vascular tumor visualized by Doppler is rarely an arteriovenous (A-V)

received

July 20, 2015 accepted after revision

October 22, 2015

published online

January 4, 2016 malformation, generally still in the quiescent stage, also known as Schobinger stage I. ${ }^{2}$ Of the high-flow lesions diagnosed in the antenatal period, the most frequent are $\mathrm{CHs}$, $\mathrm{RICH}$ being the commonest. The flow through these lesions can be extensive enough to cause high-output cardiac failure. The following case is one of an infant with $\mathrm{CH}$, RICH type diagnosed antenatally with a high-flow vascular lesion, and high cardiac output (CO) failure who required a premature delivery due to impending hydrops.
Copyright $\odot 2016$ by Thieme Medical Publishers, Inc., 333 Seventh Avenue, New York, NY 10001, USA. Tel: +1(212) 584-4662.
License terms

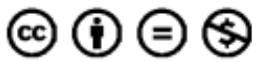

10.1055/s-0035-1570342. ISSN 2157-6998. 


\section{Case Report}

This male infant was delivered at $29+2$ weeks gestational age (GA). Antenatal scan at 20 weeks revealed a vascular mass on the right thigh, $3.6 \times 2.9 \times 2.3 \mathrm{~cm}$, composed of multiple small cysts with marked engorgement of right iliac and superficial femoral arteries (-Fig. 1A). Color Doppler images demonstrate the vascularity of the lesion (-Fig. 1B). The right iliac vein and the inferior vena cava were also markedly dilated. He was followed with serial fetal echocardiography. On subsequent scans, the lesion was increasing in size. A fetal echocardiogram done at $27+3$ weeks showed a high $\mathrm{CO}$ state with combined $\mathrm{CO}$ of approximately 1.6 multiple of median (MOM) for GA, significant cardiomegaly, and mild tricuspid regurgitation (TR). There were no signs of polyhydramnios, skin edema, ascites, or effusions at the time. At $29+0$ weeks, a fetal echocardiography demonstrated that the combined CO for GA was increasing ( $\sim 2.1 \mathrm{MOM})$ with persistent mild TR. A fetal magnetic resonance imaging at $29+0$ weeks confirmed a high-flow vascular lesion with engorged feeding vessels and a probable A-V fistula. A follow-up fetal echocardiogram at $29+2$ showed an increasing
CO ( 2.8 MOM), along with increasing TR and worsening cardiomegaly.

Although there were no overt signs of hydrops, the fetal team felt that the progression of cardiac findings would lead to imminent hydrops and a multidisciplinary decision was made with the family to deliver the fetus by cesarian section. At birth, the infant required immediate intubation and ventilation due to respiratory failure. He was noted to have a $5 \times 7 \mathrm{~cm}$ exophytic vascular lesion involving the right upper thigh. On day 2 of age, an ultrasonography with Doppler interrogation of flow in the lesion confirmed a highly vascularized tumor with high flow. At this time, the infant also presented an acute clinical deterioration with pneumoperitoneum following a bowel perforation likely due to ongoing bowel ischemia, and requiring insertion of a peritoneal drainage catheter. Computed tomography angiogram on day 4 of age allowed to better characterize the congenital hemangioma involving the right medial and anterior upper thigh with innumerable large tortuous arteries and draining veins (-Fig. 2). The main supply to the lesion was from the superficial femoral artery and the hemangioma was drained by the saphenous vein which was grossly engorged. The external

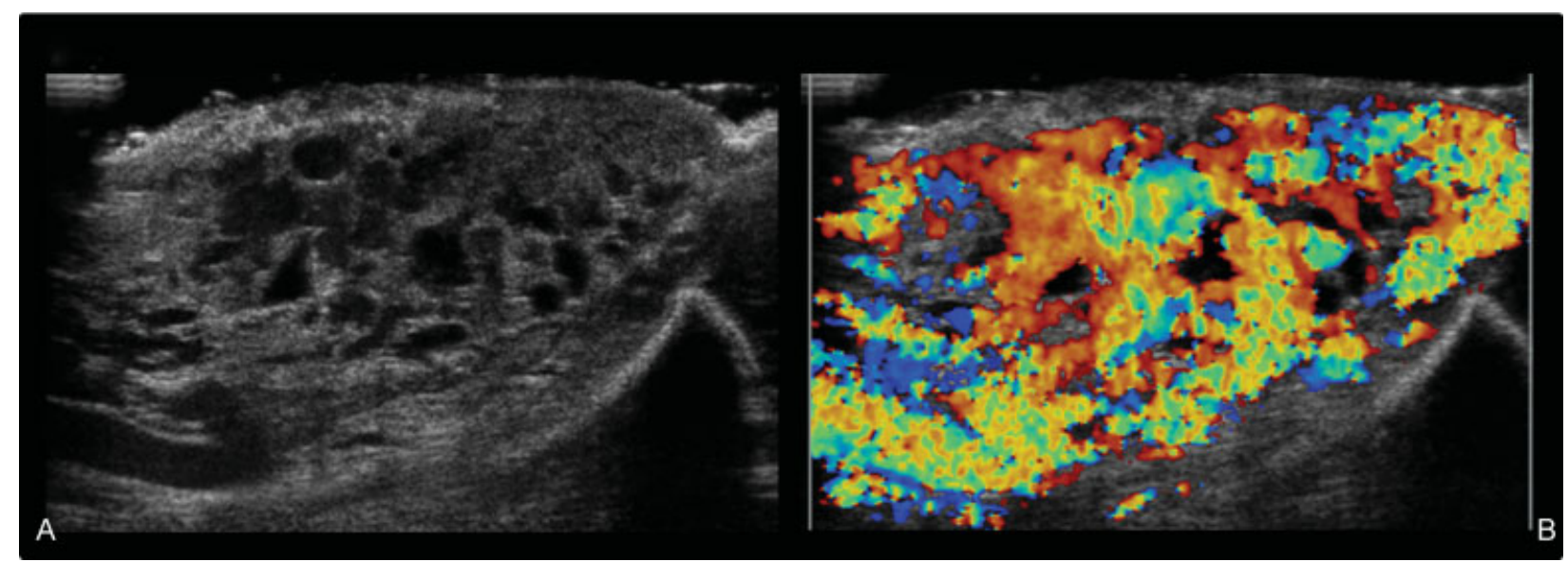

Fig. 1 (A) Selected gray scale and (B) color Doppler imaging from an ultrasound performed of the vascular mass demonstrates innumerable fluidfilled structures throughout the mass which are proven to represent profound vascularity, as reflected by the marked flow seen on (B).

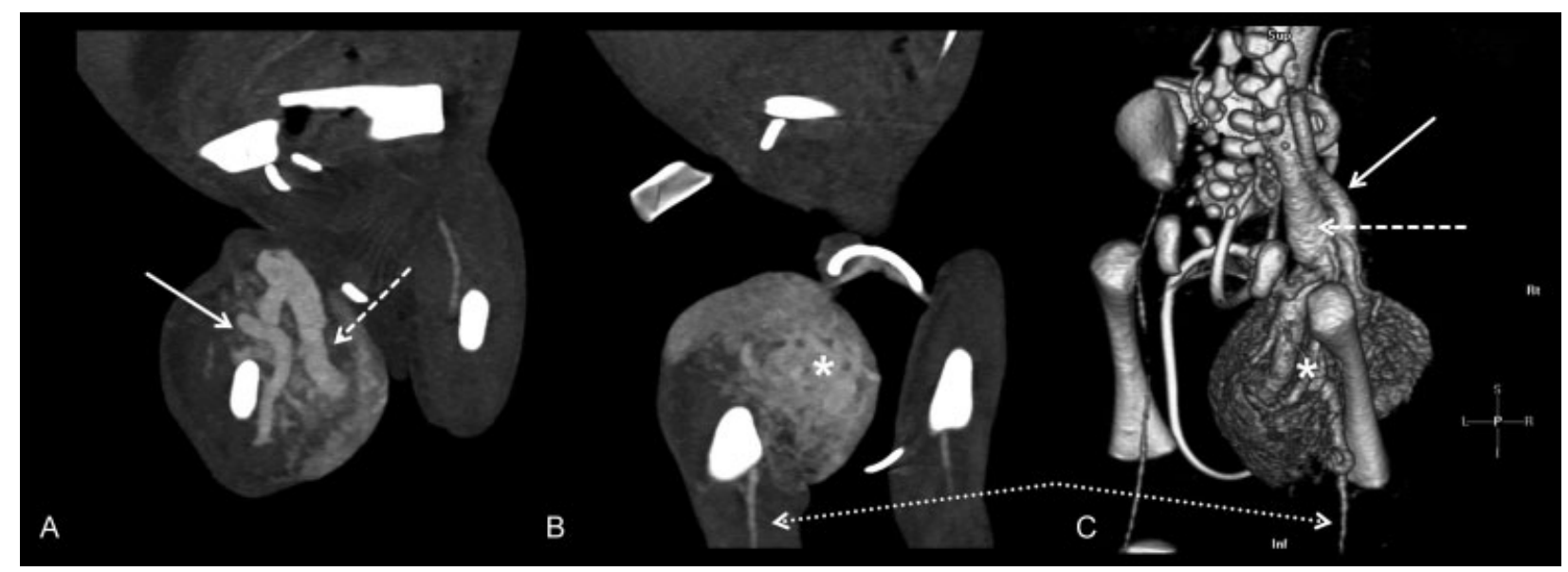

Fig. 2 (A and B) Two images from a coronal maximum intensity projection and (C) a single right posterolateral image from a three-dimensional reformat, created from a computed tomography angiogram, demonstrate massive enlargement of the right iliofemoral arterial (solid arrow) and venous (dashed arrow) circulation. The hypervascular mass representing the rapidly involuting congenital hemangiomas is well appreciated (asterisk), with the arterial circulation beyond the mass returning to a normal caliber (small dashed arrows). 
iliac and common femoral veins were also markedly dilated. His high-output cardiac state was also accompanied by high pulmonary vascular resistance (PVR) and right-to-left shunt across the patent ductus arteriosus (PDA), which was treated with an intravenous prostaglandin E1 (PGE1) infusion and inhaled nitric oxide at $20 \mathrm{ppm}$. Subsequent echocardiograms demonstrated persistent right-to-left shunting through the PDA and an estimated $\mathrm{CO}>0.5 \mathrm{~L} /$ minute during the first week of age. Also, during that time, dopamine and epinephrine were administered to maintain a normal blood pressure. Multidisciplinary consultations between neonatology, cardiology, plastic surgery, dermatology, and interventional radiology resulted in a decision to proceed with urgent embolization of the $\mathrm{CH}$. He underwent intra-arterial embolization on the 8th day of age via umbilical arterial access, with coil embolization of several of the large feeding arteries to the hemangioma (-Fig. 3) using detachable neurovascular coils (Axium; Medtronic, Brampton, Ontario, Canada) delivered through an Excelsior SL-10 microcatheter (Stryker Neurovascular, Hamilton, Ontario, Canada). Following the embolization, his CO markedly decreased, with a bidirectional shunt across the PDA indicating a partial improvement in his pulmonary pressures; however, his condition deteriorated again with increased $\mathrm{CO}$ over the next few days. Immediately postembolization, he developed a recurrence of pneumoperitoneum for which a second peritoneal drain was inserted. An exploratory laparotomy on day 11 of age revealed an isolated perforation in the distal jejunum and $2 \mathrm{~cm}$ of bowel was resected with an ileostomy performed. The surgical excision specimen was reported to show changes consistent with necrotizing enterocolitis by pathology. As the possibility of an additional procedure was considered after the first embolization, umbilical arterial access was maintained and the baby underwent repeat transarterial coil embolization on day 12 of age to further reduce the blood flow through the hemangioma ( - Fig. 4). Angiographically, marked improvement was achieved following the completion of the second embolization. Following the second embolization, his CO gradually decreased to normal values (<

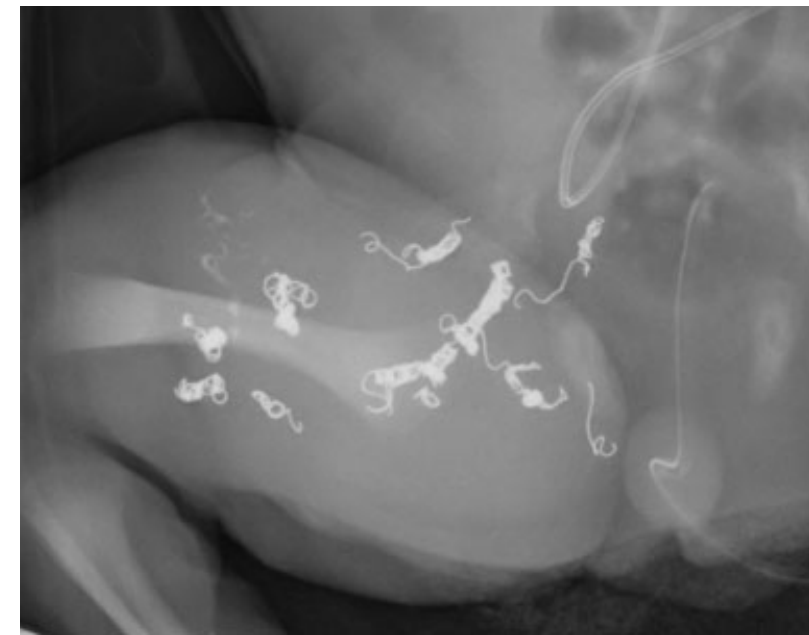

Fig. 4 Plain film imaging after the second embolization demonstrating the platinum coils used for endovascular embolization of feeding arteries to the rapidly involuting congenital hemangiomas.

$300 \mathrm{~mL} / \mathrm{kg} /$ minute). He was weaned off inhaled nitric oxide, PGE1, and inotropes in the week following the second embolization.

His highly vascular lesion with high flow also predisposed him to a state of coagulopathy and thrombocytopenia with high activated partial thromboplastin time, greater than 70 seconds (highest observed value was 105 seconds), and high D-dimer values. Following embolization, his coagulopathy improved, though he was treated with plasma infusions during that time. His platelet count showed a decreasing trend with the lowest being $94,000 \times 10^{9} / \mathrm{L}$ on day 6 of age. He remained ventilated for a total of 22 days and was subsequently discharged home postileostomy closure at 42 weeks of postmenstrual age and fully breastfed. At the 3-month corrected age (CA) follow-up visit, our patient was noted to have some leg length discrepancy, which had resolved by the 8-month CA follow-up. At 19-month CA,

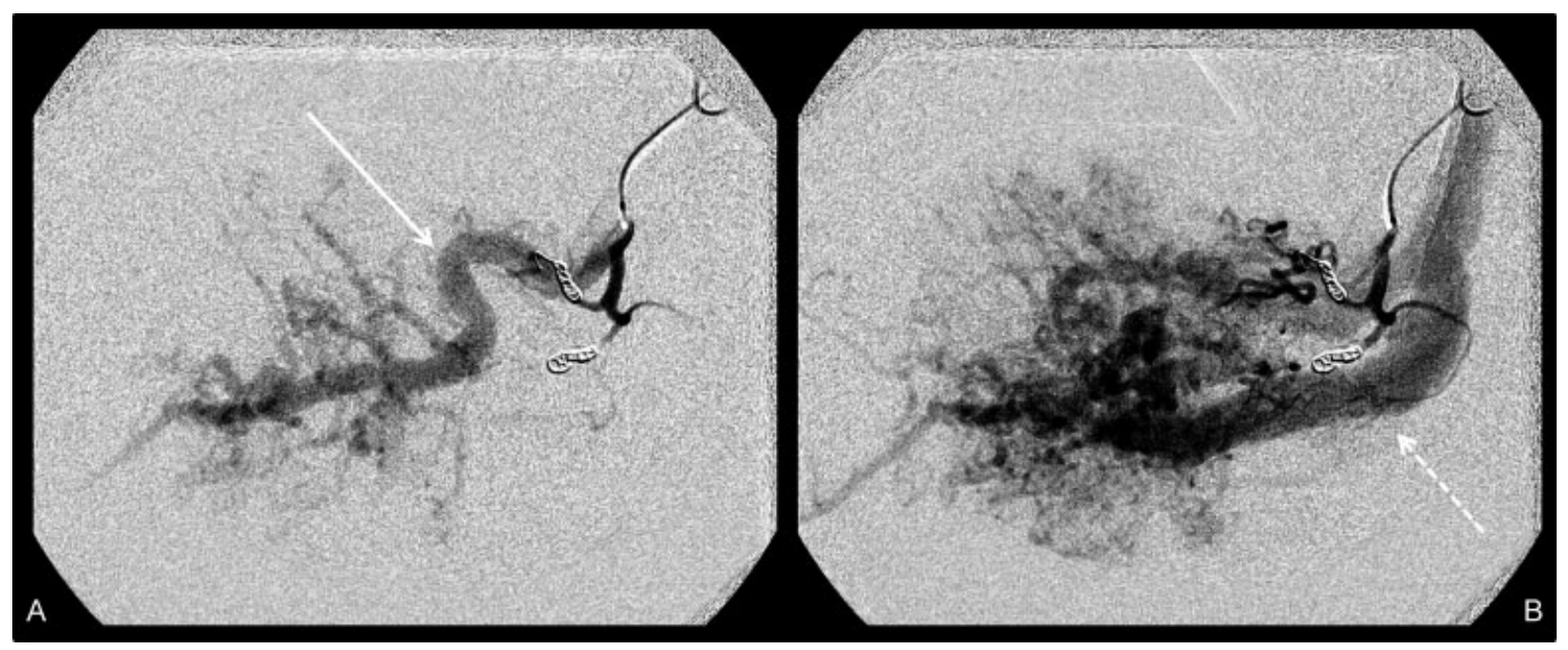

Fig. 3 (A) Early arterial and (B) parenchymal/venous phase images from the first endovascular embolization procedure demonstrate marked enlargement of the right superficial femoral artery (solid arrow), with marked lesional blush and dilation of the associated venous circulation (dashed arrow). 
our patient was noted to have equal leg lengths, although the distal and proximal thigh circumferences were $0.5 \mathrm{~cm}$ larger on the right (side of the RICH) than the left (-Fig. 5). His follow-up echocardiogram showed no evidence of pulmonary hypertension and normal left ventricular size with good function.

\section{Discussion}

This is the smallest and earliest reported case of an infant successfully managed with angioembolization for a large $\mathrm{CH}$. Based on our review of the literature, Weitz (2013) reported 17 cases of cardiac failure due to solitary cutaneous hemangioma and the majority were head and neck lesions; only two diagnosed antenatally. ${ }^{3}$ Yoon et $\mathrm{al}^{4}$ have reported the successful embolization of a cervicofacial hemangioma in a 33-week infant.

We diagnosed the congenital RICH based on clinical and radiological grounds, as an antenatally diagnosed vascular high-flow lesion is most commonly a RICH. Tufted angiomas (TAs) are rarer benign lesions which are not as aggressive and $25 \%$ of TAs are congenital, but rarely produce highoutput cardiac failure. Kaposiform hemangioendothelioma is a possible differential diagnosis with Kasabach-Merritt phenomenon, but again, it is rarer and would only be distinguished by lesion biopsy or monoclonal antibody study. Expression of the glucose transporter 1 (GLUT1) is high in proliferating and tumor cells. We did not biopsy the lesion to test for GLUT1 as our patient was very unstable. However, GLUT1 antibody detection would have been negative in both RICH and TA. The course of the lesion and presentation was thought to be very typical of RICH by our interdisciplinary team.

Our patient exhibited many complications of a large hemangioma, namely, high-output congestive heart failure, consumptive coagulopathy, and systemic hypoperfusion. The procedure of transarterial embolization carries high potential

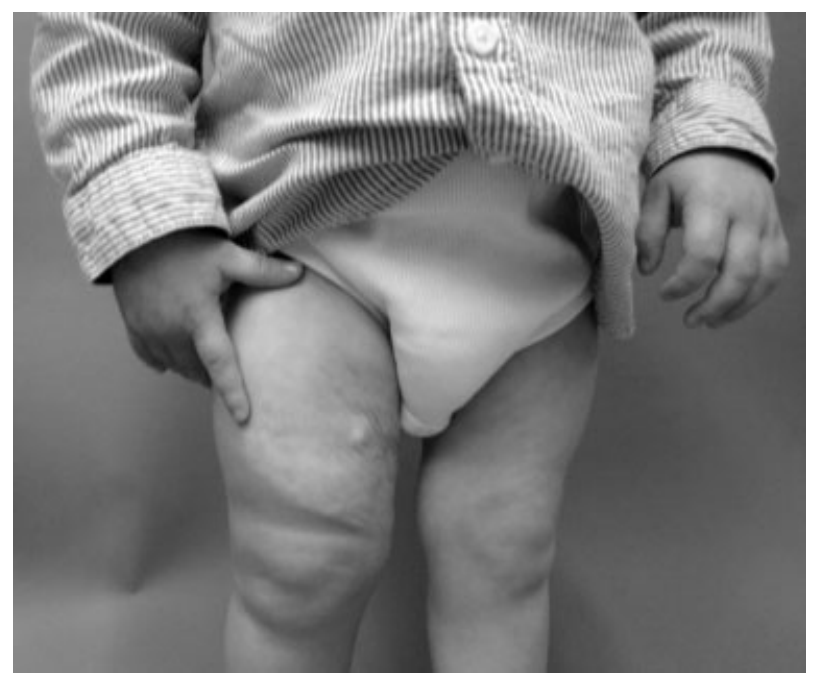

Fig. 5 Image of the resolved lesion and both limbs at follow-up visit (19-month corrected age). risks of dislodgement of emboli and is specially challenging in preterm infants due to anesthetic complications, fluid imbalance, etc. This scenario presented here illustrate several clinical dilemmas in considering the optimal time of delivery, time of embolization, and careful consideration of the balance between risks and benefits of such an intervention in a critical and hemodynamically unstable infant. Medical management of such conditions is extremely challenging and often unsuccessful. The extensive A-V shunting creates a type of "steal" phenomenon which puts other peripheral tissues at risk for infarction, such as the early jejunal perforation seen in our patient. The "steal" phenomenon also worsens the myocardial perfusion and in the face of a high-output state, this might lead to progressive myocardial ischemia. Moreover, the A-V shunt creates a low resistance systemic circuit causing a highoutput state and increased pulmonary return. This leads to an increased right-to-left shunt across the PDA and foramen ovale. High PVR and extensive right-to-left shunting across the PDA resembles the fetal circulation and the use of a PGE1 infusion may help the systemic flow and also prevent right ventricular decompensation by keeping the ductus patent. Diuretics are often used for the symptomatic management of congestive cardiac failure with inotropes and digoxin both being risky in a hyperkinetic state with myocardial ischemia. In vein of Galen malformations which have a similar physiological low resistance circuit with early congestive heart failure, the mortality was almost $100 \%$ without embolization. ${ }^{5}$ In a series of vein of Galen malformations treated with embolization, Wong et al reported an increase in mean arterial pressure following embolization and gradual progression to systemic hypertension following multiple embolizations. ${ }^{6}$ In our patient, the mean arterial pressures increased and the $\mathrm{CO}$ decreased after the embolizations, but we did not observe persistent systemic hypertension warranting treatment following embolization.

The discrepancy in measurements of the limbs at followup also illustrates the severity of the condition and may warrant a careful follow-up for early intervention to prevent gait abnormalities. Overgrowth, both axial and circumferential, has been noted in complex AV and other vascular malformations, especially involving the lower limb although this complication is unusual. ${ }^{7}$ Overall, this case underlined the paramount importance of a strategic multidisciplinary approach to uncommon, complex patients.

\section{Funding}

None.

Financial Disclosure

The authors have no financial relations relevant to this article to disclose.

Conflict of Interest

The authors have no conflict of interest to disclose. 


\section{Contributors' Statements}

Dr. Sur was the clinical fellow involved in clinical management and care of the infant. He drafted the initial article, reviewed medical records, and finalized the final formatting of the revised script.

Dr. Manraj was the involved interventional radiologist who performed the embolizations. He drafted the interventional radiology aspects and provided the radiological images.

Dr. Lim was the primary obstetrician and helped drafting the management details and discussion of the antenatal complications.

Dr. Courtemanche was the plastic surgeon who was involved in the cosmetic aspect of the lesion and was involved in follow-up assessment of the infant.

Dr. Brooks was the primary cardiologist involved in consultation and guiding the management.

Dr. Lavoie and Dr. Albersheim were the primary neonatologists actively involved in the care of the infant and reviewed, revised, and approved the final article as submitted.

Dr. Albersheim is the corresponding author.

All authors approved the final article and agree to be accountable for all aspects of the work.

\section{References}

1 Mulliken JB, Enjolras O. Congenital hemangiomas and infantile hemangioma: missing links. J Am Acad Dermatol 2004;50(6): 875-882

2 Kohout MP, Hansen M, Pribaz JJ, Mulliken JB. Arteriovenous malformations of the head and neck: natural history and management. Plast Reconstr Surg 1998;102(3):643-654

3 Weitz NA, Lauren CT, Starc TJ, et al. Congenital cutaneous hemangioma causing cardiac failure: a case report and review of the literature. Pediatr Dermatol 2013;30(6): e180-e190

4 Yoon K, Lee YJ, Park DW, Park HK, Yun HS, Lee HJ. Transarterial embolization of a cervicofacial hemangioma associated with Kasabach-Merritt syndrome in a premature neonate. J Vasc Interv Radiol 2013;24(12):1934-1936

5 Garcia-Monaco R, De Victor D, Mann C, Hannedouche A, Terbrugge $\mathrm{K}$, Lasjaunias P. Congestive cardiac manifestations from cerebrocranial arteriovenous shunts. Endovascular management in 30 children. Childs Nerv Syst 1991;7(1):48-52

6 Wong FY, Mitchell PJ, Tress BM, Dargaville PA, Loughnan PM. Hemodynamic disturbances associated with endovascular embolization in newborn infants with vein of Galen malformation. J Perinatol 2006;26(5):273-278

7 Marler JJ, Mulliken JB. Current management of hemangiomas and vascular malformations. Clin Plast Surg 2005;32(1):99-116, ix 